NASA/CR-2001-211021

ICASE Report No. 2001-19

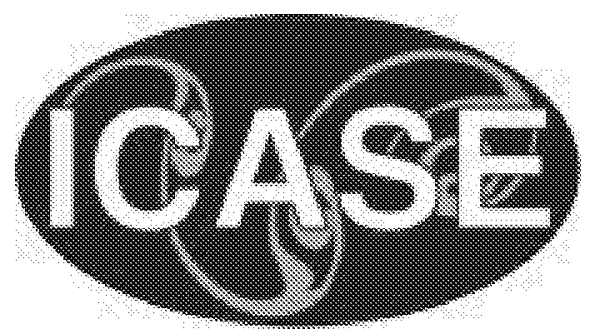

\title{
Seedless Laser Velocimetry Using Heterodyne Laser-induced Thermal Acoustics
}

Roger C. Hart

ICASE, Hampton, Virginia

R. Jeffrey Balla, G.C. Herring, and Luther N. Jenkins

NASA Langley Research Center, Hampton, Virginia 


\section{The NASA STI Program Office . . . in Profile}

Since its founding, NASA has been dedicated to the advancement of aeronautics and space science. The NASA Scientific and Technical Information (STI) Program Office plays a key part in helping NASA maintain this important role.

The NASA STI Program Office is operated by Langley Research Center, the lead center for NASA's scientific and technical information. The NASA STI Program Office provides access to the NASA STI Database, the largest collection of aeronautical and space science STI in the world. The Program Office is also NASA's institutional mechanism for disseminating the results of its research and development activities. These results are published by NASA in the NASA STI Report Series, which includes the following report types:

- TECHNICAL PUBLICATION. Reports of completed research or a major significant phase of research that present the results of NASA programs and include extensive data or theoretical analysis. Includes compilations of significant scientific and technical data and information deemed to be of continuing reference value. NASA's counterpart of peer-reviewed formal professional papers, but having less stringent limitations on manuscript length and extent of graphic presentations.

- TECHNICAL MEMORANDUM. Scientific and technical findings that are preliminary or of specialized interest, e.g., quick release reports, working papers, and bibliographies that contain minimal annotation. Does not contain extensive analysis.

- CONTRACTOR REPORT. Scientific and technical findings by NASA-sponsored contractors and grantees.
- CONFERENCE PUBLICATIONS. Collected papers from scientific and technical conferences, symposia, seminars, or other meetings sponsored or cosponsored by NASA.

- SPECIAL PUBLICATION. Scientific, technical, or historical information from NASA programs, projects, and missions, often concerned with subjects having substantial public interest.

- TECHNICAL TRANSLATION. Englishlanguage translations of foreign scientific and technical material pertinent to NASA's mission.

Specialized services that complement the STI Program Office's diverse offerings include creating custom thesauri, building customized data bases, organizing and publishing research results . . . even providing videos.

For more information about the NASA STI Program Office, see the following:

- Access the NASA STI Program Home Page at http://www.sti.nasa.gov

- Email your question via the Internet to help@sti.nasa.gov

- Fax your question to the NASA STI Help Desk at (301) 621-0134

- Telephone the NASA STI Help Desk at (301) 621-0390

- Write to: NASA STI Help Desk NASA Center for AeroSpace Information 7121 Standard Drive Hanover, MD 21076-1320 
NASA/CR-2001-211021

ICASE Report No. 2001-19

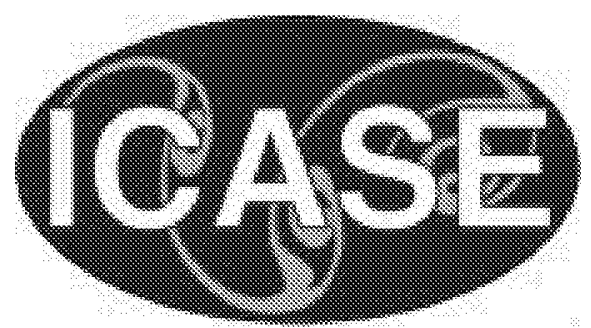

\section{Seedless Laser Velocimetry Using Heterodyne Laser-induced Thermal Acoustics}

Roger C. Hart

ICASE, Hampton, Virginia

R. Jeffrey Balla, G.C. Herring, and Luther N. Jenkins

NASA Langley Research Center, Hampton, Virginia

ICASE

NASA Langley Research Center

Hampton, Virginia

Operated by Universities Space Research Association

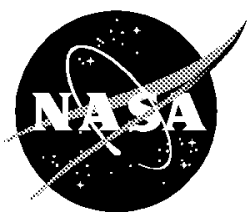

National Aeronautics and

Space Administration

Langley Research Center

Hampton, Virginia 23681-2199
Prepared for Langley Research Center under Contract NAS 1-97046 
Available from the following:

NASA Center for AeroSpace Information (CASI)

7121 Standard Drive

Hanover, MD 21076-1320

(301) 621-0390
National Technical Information Service (NTIS)

5285 Port Royal Road

Springfield, VA 22161-2171

(703) 487-4650 


\title{
SEEDLESS LASER VELOCIMETRY USING HETERODYNE LASER-INDUCED THERMAL ACOUSTICS
}

\author{
ROGER C. HART ${ }^{*}$, R. JEFFrey BALla ${ }^{\dagger}$, G. C. HERrING ${ }^{*}$, AND LUTHER N. JENKINS ${ }^{\S}$
}

\begin{abstract}
A need exists for a seedless equivalent of laser Doppler velocimetry (LDV) for use in lowturbulence or supersonic flows or elsewhere where seeding is undesirable or impractical. A compact laser velocimeter using heterodyne non-resonant laser-induced thermal acoustics (LITA) to measure a single component of velocity is described. Neither molecular (e.g. $\left.\mathrm{NO}_{2}\right)$ nor particulate seed is added to the flow. In non-resonant LITA two beams split from a short-pulse pump laser are crossed; interference produces two counterpropagating sound waves by electrostriction. A CW probe laser incident on the sound waves at the proper angle is diffracted towards a detector. Measurement of the beating between the Doppler-shifted light and a highly attenuated portion of the probe beam allows determination of one component of flow velocity, speed of sound, and temperature. The sound waves essentially take the place of the particulate seed used in LDV. The velocimeter was used to study the flow behind a rearward-facing step in NASA Langley Research Center's Basic Aerodynamics Research Tunnel. Comparison is made with pitot-static probe data in the freestream over the range $0 \mathrm{~m} / \mathrm{s}-55 \mathrm{~m} / \mathrm{s}$. Comparison with LDV is made in the recirculation region behind the step and in a well-developed boundary layer in front of the step. Good agreement is found in all cases.
\end{abstract}

Key words. LITA, laser velocimetry, laser anemometry, laser-induced thermal acoustics

Subject classification. Experimental Fluid Dynamics

1. Introduction. Laser Doppler Velocimetry is the 'gold standard' for single-point optical velocimetry methods. It is routinely applied to a great variety of flow diagnostic tasks of significance to the fluid dynamics community, and the results routinely and confidently accepted. However, situations are encountered where seeding is unacceptable or impossible. Examples include vortex cores, supersonic flows, and low-turbulence highReynolds-number cryotunnels. A seedless technique with the robustness, ease of use, and dependability of LDV would be of great utility in such circumstances. Here we present evidence that heterodyne LITA, properly implemented, is such a technique. Note that the requirement is not merely that seedless velocimetry be physically possible with LITA: rather, what is required is that dependable results be conveniently achieved by personnel who are not professional optics researchers.

" ICASE, NASA Langley Research Center, Hampton, VA 23681-2199. This research was supported by the National Aeronautics and Space Administration under NASA Contract No. NAS1-97046 while the first author was in residence at ICASE, NASA Langley Research Center, Hampton, VA 23681-2199.

${ }^{\dagger}$ AMDB, NASA Langley Research Center, Hampton, VA 23681-2199.

₹ AMDB, NASA Langley Research Center, Hampton, VA 23681-2199.

${ }^{\S}$ FPCB, NASA Langley Research Center, Hampton, VA 23681-2199. 
The use of a laser-induced grating for flow diagnostics was suggested at least as early as Eichler [1]. Cummings [24] reported the first demonstration of LITA for gas-phase thermal diffusivity and temperature measurements using seeded $\mathrm{NO}_{2}$ to enhance signal levels. Other workers have investigated the use of LITA thermometry in air [5-7], flames [8], and supersonic flows [9]. LITA velocimetry has been demonstrated using a narrow-band laser and highfinesse Fabry-Perot etalon [10], homodyne detection [11], and several approaches to heterodyne detection [12-15]. Here we present a significantly more stable approach to heterodyne detection which can form the basis for a practical, rugged, robust LITA velocimeter.

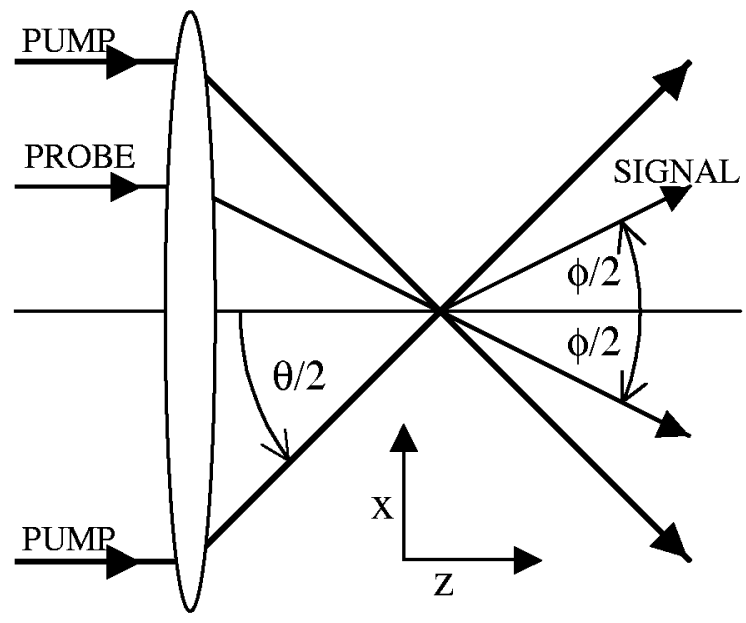

Fig. 2.1. The pump beams cross at angle $\theta$ producing an acoustic grating. Probe light incident at $\phi / 2$ is diffracted into the signal beam. $Z$ is parallel to the optic axis, while $x$ lies in the plane of the beams.

\section{Principles of LITA. A LITA measurement is a}

two-step process: a short-pulse pump laser creates acoustic waves in the medium; a long-pulse or CW probe laser beam is scattered from the moving acoustic waves by Bragg diffraction, and temperature and velocity determined by analyzing the resulting Doppler shifts. The process is reminiscent of LDV, with the acoustic waves generated by the pump laser taking the place of a seed particle. Two consequences of this 'self-seeding' are 1) measurements can be made in any portion of the flow accessible to the beams, and 2) since the data rate is determined by the firing of the pump laser and is totally independent of flow conditions, there is no velocity bias.

2.1. Pump Step. The beam from a short-pulse, high-intensity laser (e.g. Q-switched Nd:YAG) is split into two parallel, equal intensity beams with path lengths matched to within the coherence length. A lens (Fig. 2.1.) causes the beams of wavelength $\lambda_{\text {PUMP }}$ to cross at angle $\theta$ and focus at a common point. Interference fringes of period $\Lambda=\lambda_{\text {PUMP }} / 2 \sin (\theta / 2)$ form along the $\mathrm{x}$ direction at the beam crossing. In our practice, the pump light is not resonant with any absorptive transition in the medium (air); however, electrostriction creates a pressure field proportional to the intensity which is the driving term for the launch of a pair of counterpropagating acoustic waves of wavelength $\Lambda$. (See Ref. [16] for a discussion of electrostrictive laser-induced grating formation in which it is pointed out that the pump beams must be polarized normal to the crossing plane for the maximum electrostrictive effect.) The acoustic frequency in angular units measured in a frame at rest with respect to the air is then $\omega_{B}=2 \pi V_{\text {SOUND }} / \Lambda$, where $V_{\text {SOUND }}$ is the speed of sound. We estimate the intensity of the acoustic waves to be less than $100 \mathrm{~dB}$, so the waves represent a negligible perturbation to any aerodynamic phenomena.

If the pump light is resonant with an absorptive transition in the medium the efficiency of the pumping process can be greatly increased provided the absorbed energy is thermalized on a time scale short in comparison with the acoustic period. We choose not to pursue this approach because 1) it requires the introduction of a seed species into the flow, and most optically suitable seed molecules are chemically reactive or present other risks to equipment or personnel, and 2) the pump laser must be in general tuned onto the transition, which requires a tunable laser and 
possibly some means of locking the laser onto the transition. (However, both $\mathrm{I}_{2}$ and $\mathrm{NO}_{2}$ are resonant with the $2^{\text {nd }}$ Nd:YAG harmonic). Non-resonant pumping provides adequate signal levels at ambient conditions with our heterodyne detection scheme and is both simpler to implement and more broadly applicable.

2.2. Probe Step (Thermometry). The polarization induced in a medium by an incident light wave is proportional to the density. If a sinusoidal density modulation of period $\Lambda$ (the acoustic wave) is illuminated at angle $\phi / 2$ (Fig. 2.1) with light of wavelength $\lambda_{\mathrm{PROBE}}$ with $\Lambda=\lambda_{P R O B E} / 2 \sin (\phi / 2)$, the resulting polarization distribution will generate a propagating light beam at angle $\phi / 2$. This is Bragg diffraction by the acoustic wave, which hereafter we often call the acoustic grating. The requirement

$$
\frac{\lambda_{P U M P}}{2 \sin (\theta / 2)}=\Lambda=\frac{\lambda_{P R O B E}}{2 \sin (\phi / 2)}
$$

simply says the probe must be phase-matched to the grating created by the pump. For a planewave model (which assumes beams of infinite transverse extent) phase matching is a delta function in angle; for focused geometries where the gratings may be only a few tens of fringes wide phase matching is much more relaxed and requires only approximate adjustment.

In the geometry shown in Fig. 2.1 the gratings move at $\pm V_{\text {Sound }}$ with respect to the medium in the $x$ direction; additionally, there may be an $\mathrm{x}$ component $\mathrm{V}_{\mathrm{FLOW}}$ of a convective flow field. If the overlapping signal beams with Doppler shifts of $\Delta \omega_{-}=(2 \pi / \Lambda)\left(V_{F L O W}-V_{\text {SOUND }}\right)$ and $\Delta \omega_{+}=(2 \pi / \Lambda)\left(V_{F L O W}+V_{\text {SOUND }}\right)$ are incident on a detector, a modulation of the photocurrent at the difference or beat frequency $2 \omega_{\mathrm{B}}$ is observed, which, since it is proportional to the difference in the $\mathrm{x}$-component of the velocities of the two acoustic gratings, is independent of $\mathrm{V}_{\text {FLOw. }}$. If $\Lambda$ is known, speed of sound may be measured; if the composition of the medium is known, temperature may be derived. In Ref. [6] we presented an extensive laboratory study of LITA thermometry in air; to avoid errors and uncertainties in the calibration of $\Lambda$ we employed a reference cell. A small fraction of the converging pump and probe beams was split off and allowed to intersect in a cell containing air of known temperature. Comparison of the observed beat frequencies from the test and reference cells then gave the temperature in the test cell independently of $\Lambda$.

2.3. Probe Step (Velocimetry). Since two gratings moving at $\mathrm{V}_{\mathrm{FLOW}}+\mathrm{V}_{\text {SOUND }}$ and $\mathrm{V}_{\mathrm{FLOW}}-\mathrm{V}_{\mathrm{SOUND}}$ in the lab frame are created, $\mathrm{V}_{\mathrm{FLOW}}$ and $\mathrm{V}_{\text {SOUND }}$ may be independently determined. If a beam (termed the local oscillator) at the frequency of the probe is introduced collinear to the signal beams, the time-varying intensity on the detector is

$$
\begin{aligned}
I(t) \propto & \sqrt{I_{L O} I_{S I G N A L}}\left(\cos \left(\Delta \omega_{+} t\right)+\cos \left(\Delta \omega_{-} t\right)\right) \\
& +I_{S I G N A L} \cos \left(2 \omega_{B} t\right)
\end{aligned}
$$

where $\mathrm{I}_{\mathrm{LO}}$ and $\mathrm{I}_{\text {SIGNAL }}$ are the intensities of the local oscillator and signal beams. Spectral analysis (e.g. discrete Fourier transform) of a real-valued time series cannot retrieve $\Delta \omega_{+}$or $\Delta \omega_{-}$, but only $\left|\Delta \omega_{+}\right|$and $\left|\Delta \omega_{-}\right|$. If $\mathrm{V}_{\mathrm{FLOW}}<$ $\mathrm{V}_{\text {SOUND }}$, then

$$
\left|V_{F L O W}\right|=\frac{1}{2}\left(\frac{\Lambda}{2 \pi}\right)|| \Delta \omega_{+}|-| \Delta \omega_{-}||
$$


and

$$
V_{\text {SOUND }}=\frac{1}{2}\left(\frac{\Lambda}{2 \pi}\right)\left(\left|\Delta \omega_{+}\right|+\left|\Delta \omega_{-}\right|\right) .
$$

Note that Mach number

$$
M=\frac{\left|V_{\text {FLOW }}\right|}{V_{\text {SOUND }}}=\frac{|| \Delta \omega_{+}|-| \Delta \omega_{-}||}{\left|\Delta \omega_{+}\right|+\left|\Delta \omega_{-}\right|}
$$

is found independently of $\Lambda$. For measurements in an isothermal, incompressible flow, $V_{\text {SOUND }}$ may be determined

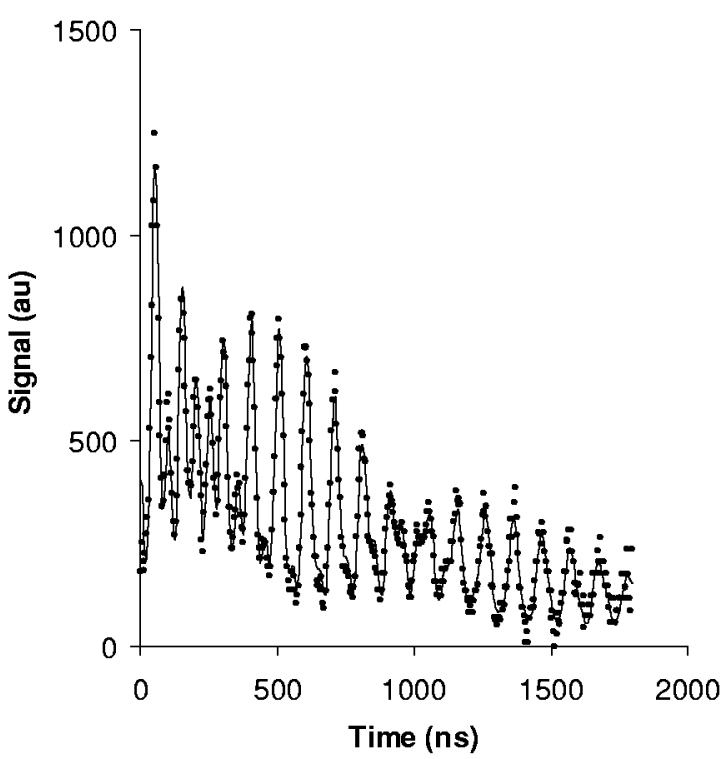

Fig. 2.2. Single-shot heterodyne LITA waveform (points) and fit.

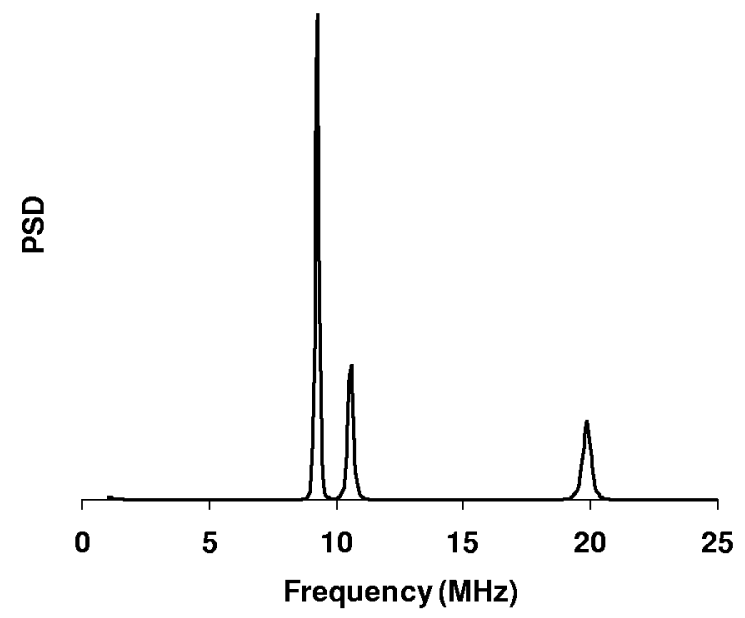

Fig. 2.3. Prony's method fit to data of Fig. 2.2 shown in frequency domain. $P S D$ is power spectral density. from the known flow temperature and $V_{\text {FLOW }}$ found without other calibration. If the flow is not isothermal, a reference cell may be used or $\Lambda$ may be calibrated by measuring $2 \omega_{\mathrm{B}}$ at known temperature.

In Fig. 2.2 is shown a digital oscilloscope trace of a single heterodyne LITA shot as well as the results of a fit using Prony's method, which is discussed below. The decay of the signal with time is due to acoustic absorption and, to a much smaller extent in the data shown, by motion of the grating out of the probe beam. Acoustic absorption is described by Beer's law $I=I_{0} \exp \left(-2 \alpha V_{\text {SOUND }} t\right)$ with the amplitude absorption coefficient $\alpha \propto f^{2} / P$, where $\mathbf{f}$ is the frequency of the sound wave and $\mathrm{P}$ is pressure. For air at atmospheric pressure $\alpha / \mathrm{f}^{2}=1.37 \times 10^{-11} \mathrm{~s}^{2} / \mathrm{m}$ [17].

In Fig. 2.3 we show the results of the fit to the data in Fig. 2.2 plotted in the frequency domain. The small peak at $\sim 20 \mathrm{MHz}$ is at frequency $2 \omega_{\mathrm{B}}$ which depends only on the speed of sound. It results from the beating together of the signal beams from the two counterpropagating acoustic gratings. The two peaks near $10 \mathrm{MHz}$ result from the beating together of the local oscillator beam and the two signal beams individually. If the flow velocity is zero these two peaks coalesce at $\omega_{\mathrm{B}}$. As flow velocity increases their separation widens.

The scheme described above (which we refer to as the central fringe method) was evaluated by us in a series of laboratory experiments in a free jet which gave good agreement with pitot-static measurements over the range 20 $150 \mathrm{~m} / \mathrm{s}$ [15]. However, performance at lower velocities was 
poor, due to the inability to accurately determine $\left|\Delta \omega_{+}\right|-\left|\Delta \omega_{-}\right|$from noisy data when the two peaks (Fig. 2.3) were not well resolved spectrally. Worse, alignment of the local oscillator with the signal beams was quite critical: even with sturdy, high quality mounts and massive optical tables an excessive amount of 'tweaking' was required before each data collection run. While adequate for demonstrating the principle, this was clearly not an approach that could form the basis for a robust, set-and-forget diagnostic.

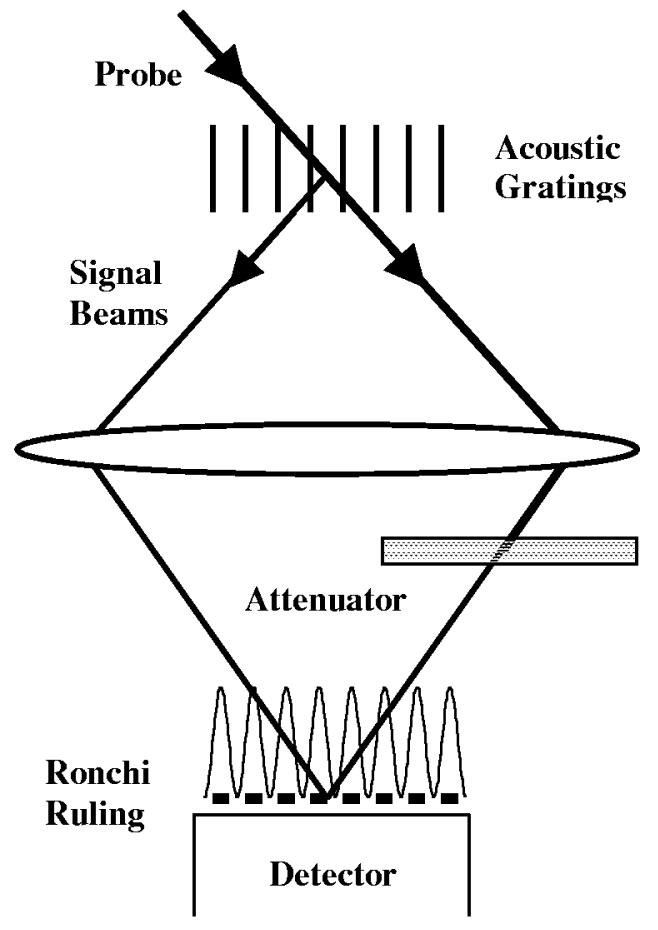

Fig. 2.4. Schematic of grating demodulation scheme.
2.4. Grating Demodulation. Consider the arrangement shown in Fig. 2.4. A lens causes the probe and signal beams to refocus and recross with (for simplicity) unit magnification. An attenuator placed in the probe beam reduces its intensity to $\mathrm{I}_{\mathrm{LO}}$. If the acoustic gratings were motionless, and the signal beams thus at the same frequency as the local oscillator beam, stationary interference fringes of spatial period $\Lambda$ would be formed. Since the signal beams are Doppler-shifted by the motion of the acoustic gratings, the fringes move at the speed of the acoustic gratings (in the case of unit magnification). One is in effect imaging the sound waves. A broad-area detector placed at the plane of the crossing would show no modulation, since the intensity integrated over the area of the beams is constant with time. However, if a series of slits of spacing $\Lambda$ (a Ronchi ruling, for instance) is placed immediately in front of the detector the modulation is recovered just as if the signal and local oscillator beams were collinear. Alternatively, one may consider the diffractive nature of the Ronchi ruling. It can easily be shown that a

ruling of spacing $\Lambda$ illuminated at $\pm \phi / 2$ with $\Lambda=\lambda_{P R O B E} / 2 \sin (\phi / 2)$ will diffract the first order of one beam parallel to the zero'th diffraction order of the other: the Ronchi ruling (or a phase grating) can serve as a diffractive beam mixer, automatically producing collinear probe and signal beams. We evaluated both approaches, by placing a multi-mode optical fiber (coupled to a detector) immediately behind the ruling, and by placing the fiber with a freespace to fiber coupler some distance away from the ruling so only one of the several diffracted sets of beams would enter the fiber. We also evaluated a diffractive phase grating in place of the Ronchi ruling. No approach was found conspicuously superior to any other.

The advantage of this scheme, which we refer to as grating demodulation, is that it forms a common-path interferometer. With the exception of the attenuator placed in the probe beam, every optical element in the receiver is common to both signal and probe paths, and so the system is insensitive to vibration or misalignment of the optics. The central-fringe method, on the other hand, forms a divided-path interferometer and is extremely sensitive to vibration and misalignment. (The method described in Ref. [18] is also common path, but is unsuitable for 
applications where the beams must be focused.) In our experience the grating method requires little or no adjustment after initial alignment. It thus forms the basis for a robust diagnostic instrument.

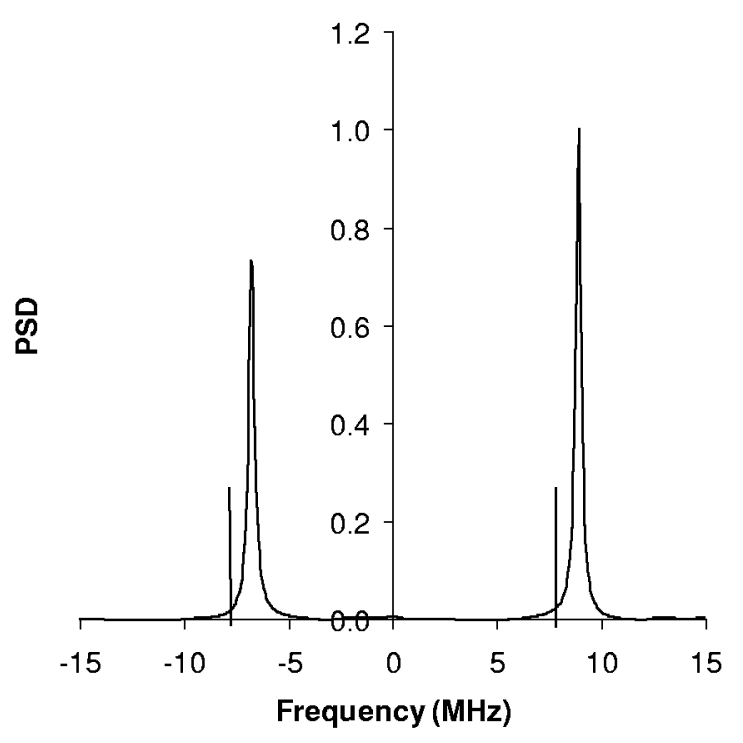

Fig. 2.5. Spectral content of LITA shot taken using quadrature detection. The vertical lines indicate $\pm \omega_{B}$.
2.5. Quadrature Detection. If the Ronchi ruling in Fig. 2.4 is translated transverse to the optical axis in the direction normal to the slits, the temporal phase of the signal modulation will shift just as if the phase of the local oscillator beam had been changed. Use of a suitable beamsplitter and two Ronchi rulings shifted by $\Lambda / 4$ with respect to one another, as well as two detectors, conveniently allows heterodyne detection of the signal with effectively two phases of the local oscillator differing by $\pi / 2$ : this is commonly termed quadrature detection. One recovers the time variation of both the amplitude and the phase of the signal beam. This modestly improves the effective signal-tonoise ratio, since the two quadrature signals are independent. More importantly, it allows direct determination of the direction of flow velocity. Most significantly, it greatly improves the resolution of measurements made at low velocities.

As noted above, spectral analysis of real-valued data can retrieve only the absolute value of the frequency, so $\mathrm{P}(\omega)=\mathrm{P}(-\omega)$, where $\mathrm{P}(\omega)$ is the power spectral density at frequency $\omega$. However, analysis of complex-valued data $\mathrm{I}(\mathrm{t})=\mathrm{I}_{0}(\mathrm{t})+i \mathrm{I}_{90}(\mathrm{t})$, where $\mathrm{I}_{0}$ and $\mathrm{I}_{90}$ are the two quadrature components, retrieves the signed value of the frequency. In Fig. 2.5 we show the spectral content of a single LITA shot taken in this way. If $\mathrm{V}_{\text {FLOW }}=0$, the two peaks will occur at $\pm \omega_{\mathrm{B}}$. Since frequency is the time rate of change of phase, and phase in this case is proportional to the negative of the distance probe laser - acoustic grating - detector, we may identify the spectral peak at positive frequency with the acoustic grating that is moving towards the laser and detector (phase increasing with time), and the negative frequency peak with the grating that is moving away (phase decreasing with time). If convective flow is present, the spacing between the two peaks will remain equal to $2 \omega_{\mathrm{B}}$ (assuming temperature remains constant) but the peaks will shift to the left or right of $\pm \omega_{\mathrm{B}}$ in Fig. 2.5. That is

$$
V_{F L O W}=\frac{1}{2}\left(\frac{\Lambda}{2 \pi}\right)\left(\Delta \omega_{+}+\Delta \omega_{-}\right)
$$

and

$$
V_{\text {SOUND }}=\frac{1}{2}\left(\frac{\Lambda}{2 \pi}\right)\left(\Delta \omega_{+}-\Delta \omega_{-}\right)
$$

where $\mathrm{V}_{\text {FLOW }}$ is now a signed quantity giving flow direction and magnitude. The relation between complex-valued and real-valued data can be seen by simply folding the spectrum of Fig. 2.5 about $\omega=0$ : this produces two peaks 
symmetrically placed about $\omega_{\mathrm{B}}$ as seen in Fig. 2.3. The improvement in low-velocity resolution with complexvalued data is due simply to the fact that the peaks no longer overlap.

2.6 Data Reduction. A discrete Fourier transform can be used to retrieve the spectral content of either real or complex-valued LITA signals. However, significantly better precision is available by taking advantage of the fact that one knows in general what the signal should look like: that is, one can fit a physical model to the observed data. A reasonable model for the LITA signal is a sum of damped complex exponentials of the form

$$
I(t)=\sum_{k=1}^{3} A_{k} \exp \left(-\beta_{k} t\right) \exp \left(-i \omega_{k} t\right)
$$

where $\omega_{1,2,3}=\omega_{+}, \omega_{-}, 2 \omega_{B}$. This model in general requires use of a non-linear fitting algorithm (e.g. LevinburgMarquardt). However, Prony's method allows fitting of complex exponentials using a linear fitting algorithm and is thus much more stable and much faster [19]. The order (number of complex exponentials to fit) was generally set to sixty to allow the algorithm ample resources to model the noise on the waveform.

3. Description of Instrument. The one-component LITA velocimeter consists primarily of transmitter and receiver modules which contain the lasers and all the optics, plus ancillary equipment such as laser power supplies and a rack for the electronics and computer. The transmitter and receiver modules are both approximately 2' long by 1' wide by 16" high and weigh less than $100 \mathrm{lbs}$. each. They can be carried and installed by two people.

The pump is a $400 \mathrm{~mJ} /$ pulse, $20 \mathrm{~Hz}$ repetition rate flash-lamp pumped, Q-switched Nd:YAG laser with output at $1064 \mathrm{~nm}$. The laser head is sealed and employs hard-mounted (non-adjustable) optics. The probe laser is diode pumped and provides $5 \mathrm{~W}$ of CW $532 \mathrm{~nm}$ light. The probe laser head is also sealed and non-adjustable. Both lasers operate from $115 \mathrm{~V}$ mains supplies and neither requires an external cooling water supply. The pump and probe laser heads are demountable from the transmitter module so the lasers may be transported separately.

The pump and probe beams are focused and crossed by a $750 \mathrm{~mm}$ focal length plano-convex lens. The probe focal spot diameter was measured to be about $300 \mu \mathrm{m}$, while the pump spot diameter was $1.5 \mathrm{~mm}$ giving a pump intensity of $\sim 2.4 \times 10^{9} \mathrm{~W} / \mathrm{cm}^{2}$. The pump crossing angle was $1.4^{\circ}$ giving $\omega_{\mathrm{B}} \approx 7.8 \mathrm{MHz}$. The large pump spot size is due to the multiple transverse mode output of the pump laser, and in fact a 1.5:1 telescope was employed to enlarge the pump beam before splitting and focusing to reduce the focal spot diameter.

In addition to the grating demodulation optics, the receiver contains the optics for the Focal Plane Camera (FPC). A very small fraction of the pump and probe beams is split off and brought to a focus; this focus is then reimaged onto a CCD array using a microscope objective. The purpose of this is to allow real-time monitoring of the relative positions of the probe and the two pump beams at the beam crossing point. Slight adjustments in the beam positions need to be made from time to time, especially to the pump beam overlap due to the multiple transverse mode output of the pump laser. Being able to inspect the beam positions as well as the resulting signal greatly eases this process.

4. Tunnel and Model. The NASA Langley Research Center Basic Aerodynamics Research Tunnel (BART) is an open-loop facility with a test section $1 \mathrm{~m}$ wide by $0.7 \mathrm{~m}$ high by $3 \mathrm{~m}$ long. A single-stage motor-driven fan produces flow velocities up to $65 \mathrm{~m} / \mathrm{s}$ with turbulence levels measured at less than $0.1 \%$. Large float-glass windows 


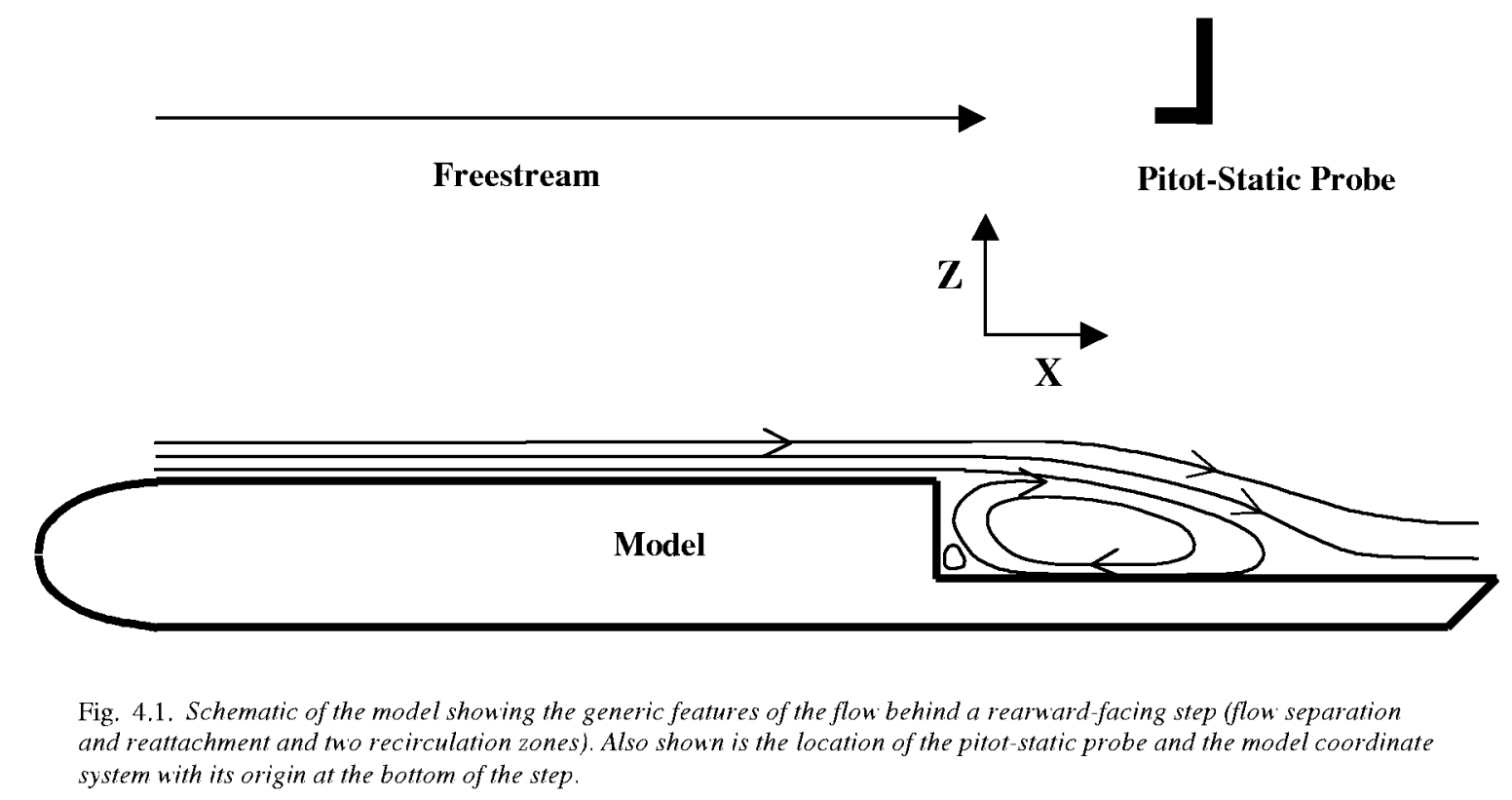

were installed on the sides of the test section for this test. The $1.5 \mathrm{~m}$ long model (Fig. 4.1) was mounted at an angle of attack of $1.6^{\circ}$. A pitot-static probe was mounted approximately $0.4 \mathrm{~m}$ above the model to measure the freestream velocity. A thermocouple mounted in the freestream downstream of the pitot tube provided real-time measurements of air temperature for calibration. A large, nested-cube three-axis traverser surrounds the test section. The LITA transmitter and receiver were mounted on the innermost cube on opposite sides of the tunnel. Calibration of the traverser coordinate system with respect to the model was done with burn paper and the probe laser.

The step height $\mathrm{h}$ of the rearward-facing step was $3.8 \mathrm{~cm}$ and the shelf behind the step was approximately $12 \mathrm{~h}$ long. Shown schematically in Fig. 4.1 is the flow behind the rearward-facing step, including flow separation and reattachment and primary and secondary recirculations. Also shown in Fig. 4.1 is the coordinate system used in the discussion that follows. The origin was chosen as the bottom corner of the step with $\mathrm{x}$ parallel to the plate and $\mathrm{z}$ normal to the plate.

5. Experimental Results. Installation and initial operation of the LITA velocimeter at BART took approximately two days; after taking some good quality initial data several days were spent making modifications to enhance the stability and usability of the instrument. Thereafter data taking proceeded uninterrupted by anything more than minor alignment adjustments. We find this quite promising considering that this was a completely new design of instrument on its first trip out of the laboratory. For comparison, installation of an LDV system by a highly experienced operator took approximately 1 1/2 days.

5.1. Comparison with Pitot-Static Tube. For comparison with velocities determined by the pitot-static probe the LITA measurement volume was positioned approximately $1 \mathrm{~cm}$ in front of the probe. The tunnel was stabilized at a series of speeds up to $55 \mathrm{~m} / \mathrm{s}$. At each speed 375 individual shots were taken, this number being determined by the storage capacity of the digital oscilloscope. This process was repeated on five days during the course of our test period. The results are summarized in Figs. 5.1 through 5.3. 


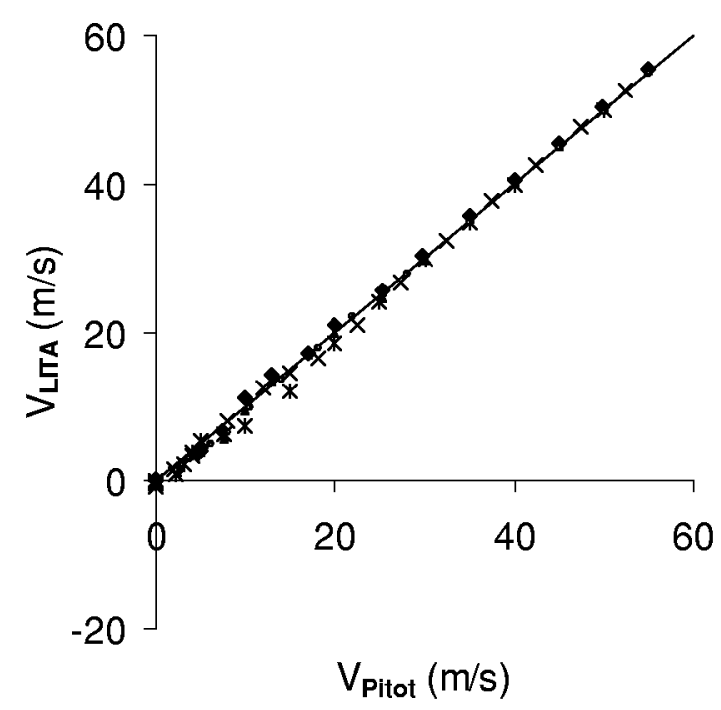

Fig. 5.1. Plot of velocity measured by LITA $V_{L I T A}$ is. velocit measured by pitot-static probe $V_{\text {PITOT }}$ in freestream. Datafrom five days is shoun in differing symbols.

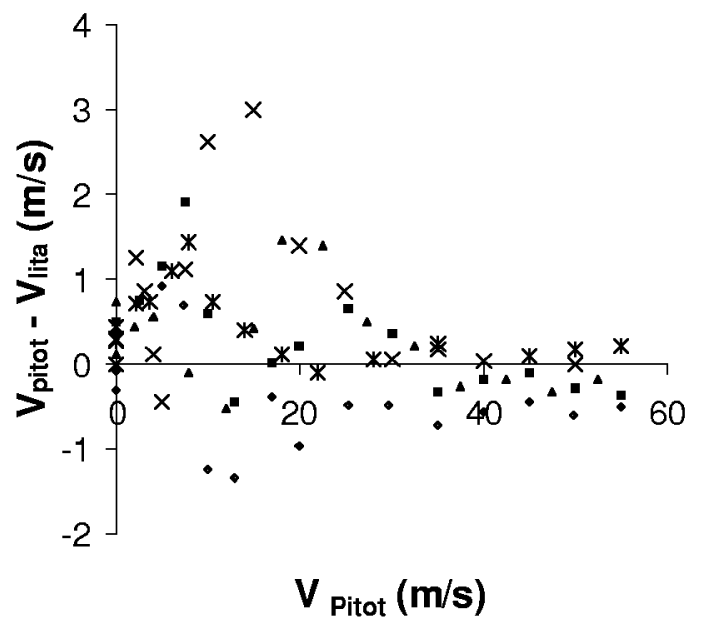

Fig. 5.2. Error in LITA velocity measurement is. freestream velocity. Data from five days is shown in differing symbols.

Figure 5.1 plots the mean value $V_{\text {LITA }}$ of each 375 shot data record vs. the flow velocity as determined by the pitot tube $V_{\text {PाтOT }}$, while Fig. 5.2 shows the error $V_{\text {LTTA }}-V_{\text {PाTOT }}$ vs. $V_{\text {РाTOT }}$. No error bars are shown, as the standard error of the mean would be little larger than the plot symbols in Fig. 5.2. In other words, the errors visible at velocities below $30 \mathrm{~m} / \mathrm{s}$ are statistically significant. At zero velocity and for $\mathrm{V}>30 \mathrm{~m} / \mathrm{s}$ there is good run-to-run consistency and good agreement between LITA and the pitot tube, with all points showing errors of less than \pm 0.5 $\mathrm{m} / \mathrm{s}$. In the region $0<\mathrm{V}<30 \mathrm{~m} / \mathrm{s}$ there is substantially more run-to-run variation. We have determined that the bulk of the error here was due to variation of the phase angle between the two quadrature components. If this angle is not

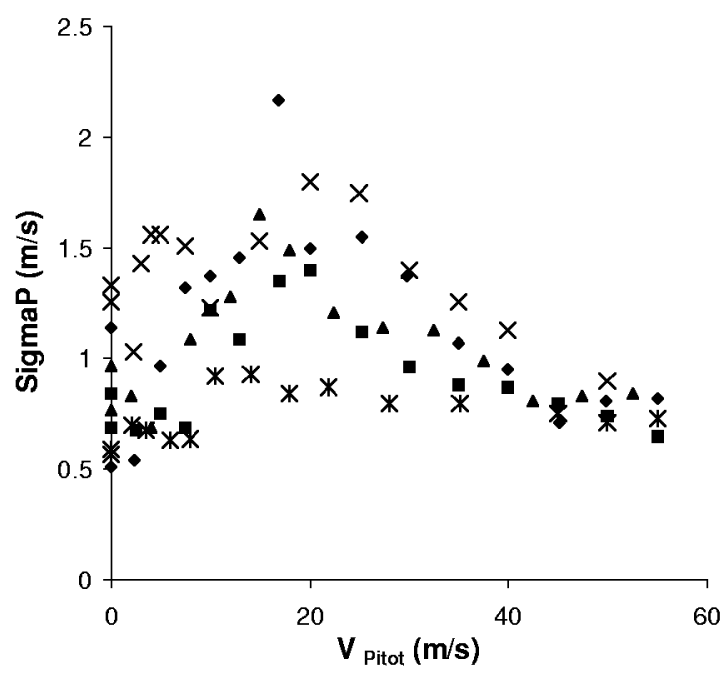

Fig. 5.3. Population standard deviation SigmaP of each of the mean values shown if Figs. 5.1 and 5.2 . equal to $\pi / 2$ the effect is to mix the real and imaginary components of the signal. For an intermediate range of flow velocities this can result in a systematic error.

Figure 5.3 shows the population standard deviation

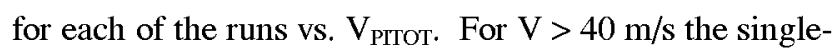
shot $1 \sigma$ uncertainty is $<1 \mathrm{~m} / \mathrm{s}$, but run-to-run variation again increases at lower flow velocities. One source of this variation was day-to-day differences in the placement of the large pump spot with respect to the probe beam, resulting in changes of the relative heights of the two peaks in the spectrum due to the differential motion of the two acoustic gratings. A simple change to our software to weight the two peaks according to their area in the calculation of $V_{\text {FLOW }}$ and $V_{\text {SOUND }}$ somewhat improved the results. A better solution is likely an adaptive, real-time 


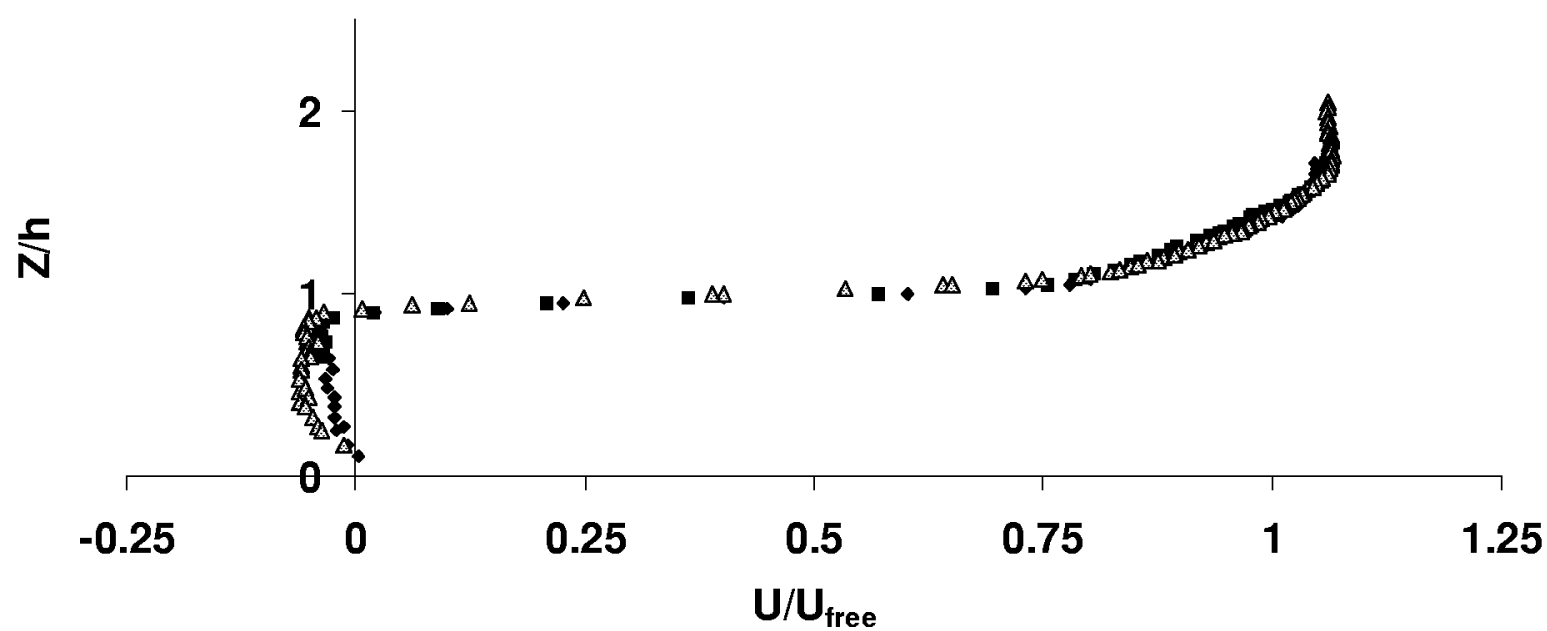

Fig. 5.4. Mean velocity profiles at $x / h=0.52$ taken using LITA (dark symbols) and LDV (gray triangles). The LDV data is not corrected for velocity bias.

adjustment of the positioning of the pump spot to optimize the 'aim-off' of the pump.

5.2. Comparison with LDV. After completion of our program of LITA measurements the LITA apparatus was removed and a two-component LDV system was installed. Certain flow profiles made with LITA were then repeated using LDV under identical run conditions, the results of which are shown in Figs. 5.4 - 5.6. The tunnel speed was $45 \mathrm{~m} / \mathrm{s}(\mathrm{M}=0.13)$ for all measurements, and data records of 375 shots (LITA) and 500 particles (LDV) were averaged for each data point.

In Figs. 5.4 and 5.5 we show LITA and LDV profiles in the $\mathrm{z}$ (normal to plate) direction just downstream of

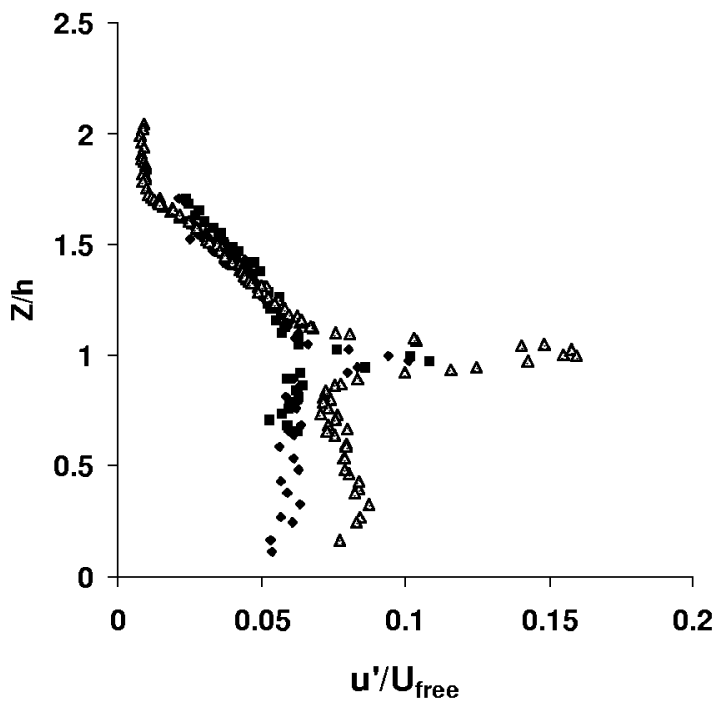

Fig. 5.5. RMS velocity profiles at $x / h=0.52$ taken using LITA (dark symbols) and LDV (gray triangles). the step at $\mathrm{x} / \mathrm{h}=0.52$, with Fig. 5.4 showing streamwise velocity $U$ normalized by the freestream velocity $U_{F R E E}$, while Fig. 5.5 shows the population standard deviation $u$ ' (or 'rms') similarly normalized. Both LITA and LDV profiles show a strong shear layer at $\mathrm{z} / \mathrm{h} \sim 1$ as expected with recirculation (forward flow) for $\mathrm{z} / \mathrm{h}<0.9$. Agreement between LITA and LDV above the shear layer is excellent. Note that the LITA data comprises two runs taken several days apart. However, below the shear layer in Fig. 5.4, and from the shear layer down in Fig. 5.5 there is a significant discrepancy between LITA and LDV, which is due to uncorrected velocity bias in the LDV results. As one would expect, no velocity bias is present above the shear layer where flow and seeding are uniform. In the shear and recirculation regions, where the flow is not 


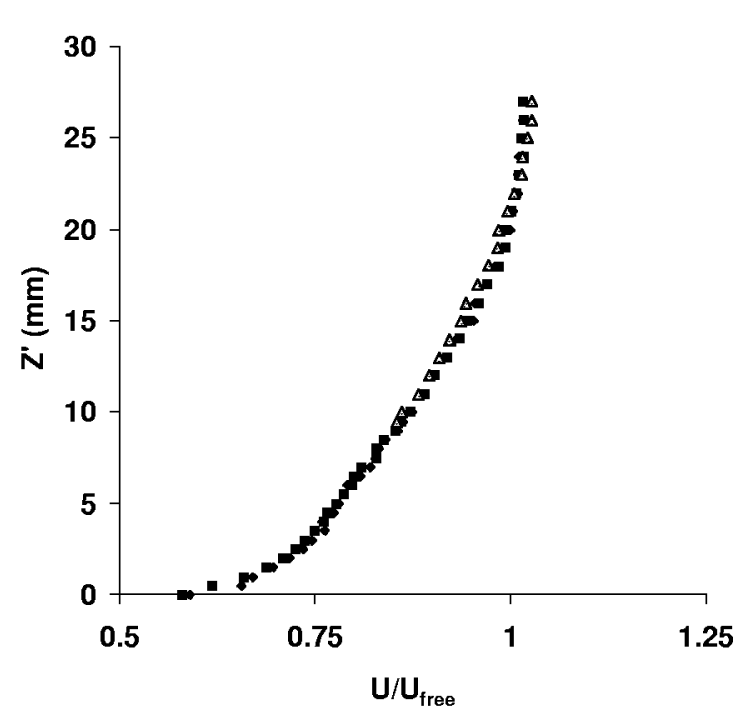

Fig. 5.6. Mean velocity profiles of a turbulent boundary layer taken with LITA (dark symbols) and LDV (gray triangles). Here $z$ ' is height above the top surface of the model.

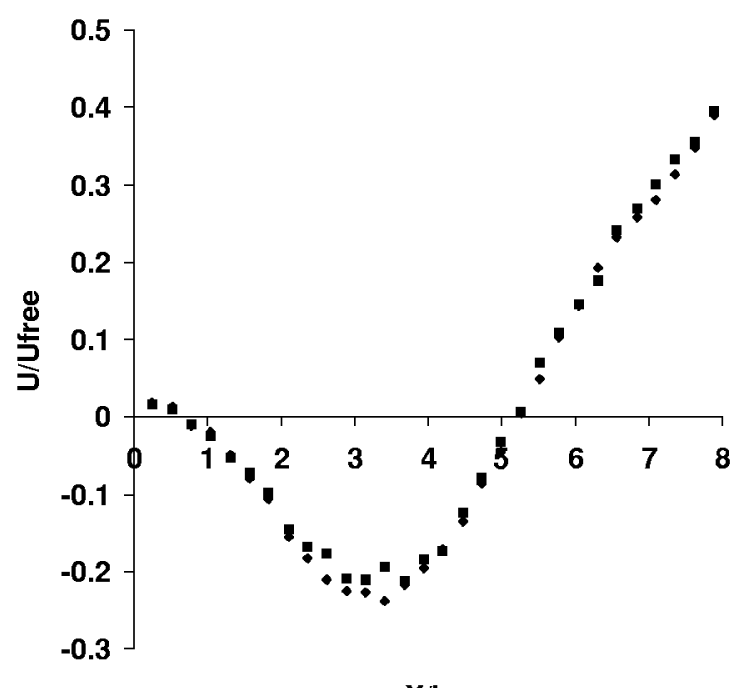

$\mathbf{X} / \mathbf{h}$

Fig. 5.7. Mean velocity profiles taken parallel to the plate behind the rearward-facing step showing three regions of the flow. LITA runs from two days are shown in different symbols. uniform, the velocity bias results in overestimation of both the average and rms velocities by LDV, with the discrepancy larger for the rms.

In Fig. 5.6 we show LITA and LDV profiles of a fully-turbulent boundary layer (Reynolds number $\mathrm{R}_{\mathrm{L}} \sim 2 \mathrm{X}$ $10^{6}$ based on the length from the front of the model) on the top surface of the model just ahead of the step. Here $z$ ' is height above the surface of the model. Two LITA profiles are shown, taken several days apart. Again there is excellent agreement between LITA and LDV as well as between the two LITA runs.

5.3. Other Results. Figure 5.7 shows the results of two LITA profiles of U/U $\mathrm{U}_{\mathrm{FREE}}$ taken parallel to the plate at a height $\mathrm{z} / \mathrm{h}=0.09$; the two data sets were taken two weeks apart and show excellent agreement. Three flow regions can be seen: the region of streamwise flow downstream of the reattachment; the primary recirculation region with upstream flow peaking at about $-0.25 \mathrm{U}_{\mathrm{FREE}}$; and, just behind the step, a region of streamwise flow which is a weak secondary recirculation region. Note that the two points in the secondary recirculation are at $\sim 1 \%$ and $2 \%$ of $\mathrm{U}_{\mathrm{FREE}}$ or about 0.5 and $1 \mathrm{~m} / \mathrm{s}$ respectively.

In Fig. 5.8 we present part of a grid of data points taken in the recirculation region. The data is taken at uniformly spaced stations downstream of the step and the velocities scaled on the graph so that $\mathrm{U}_{\text {FREE }}$ is the distance from one station to the next. One sees the shear layer broaden and move lower as $\mathrm{x} / \mathrm{h}$ increases, while the upstream component of the recirculation velocity increases.

\section{Summary and Conclusion. A compact,} portable, single-component heterodyne LITA velocimeter was constructed and used to measure velocities in the freestream, behind a rearward-facing step, and in a turbulent boundary layer at the NASA Langley Research Center BART facility. Overall agreement with pitot-static probe measurements and with LDV was excellent. Repeated measurement of various flow profiles shows excellent stability. Use of a thermocouple in the incompressible $(\mathrm{M}=$ 0.13 ) flow allowed these accurate and repeatable results to be obtained without calibration. Infrequent adjustments 


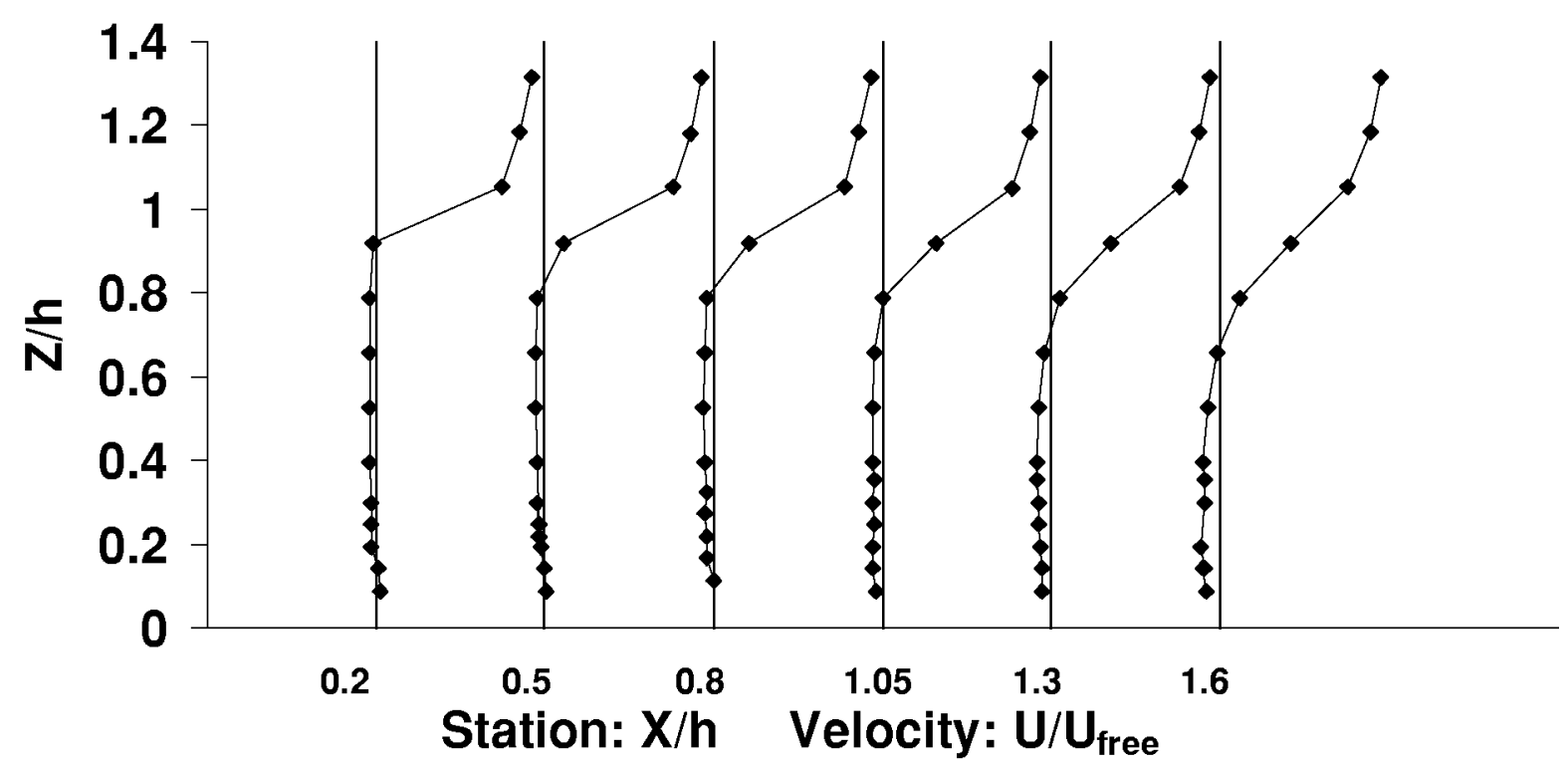

Fig. 5.8. Grid of data points showing mean velocities in shear layer and recirculation region behind the step. The velocity is scaled so that the distance on the graph between data stations (indicated by vertical lines) is equal to the freestream velocity. Thus the vertical line at station $x / h=0.5$ indicates $0 \mathrm{~m} / \mathrm{s}$ for the data set taken at $x / h=0.5,+U_{F R E E}$ for data taken at $x / h=0.2$, and $U_{F R E E}$ for data taken at $x / h=0.8$. The lines connecting data points taken at a given station are for clarity only.

to this first-generation instrument were required, but did not constitute an excessive workload; modest additional development should allow the construction of an instrument that requires levels of effort and skill similar to LDV.

The authors gratefully acknowledge the patient and untiring assistance of Mark Fletcher in the execution of this work. We also gratefully acknowledge the invaluable assistance of Richard White in the preparation of the model and operation of the tunnel, and Timothy Hepner for setting up the LDV apparatus.

\section{REFERENCES}

[1] H. J. EICHLER, P. GUNTER, D. W. POHL, Laser-Induced Dynamic Gratings, Springer-Verlag (1986).

[2] E. B. CUMMINGS, Laser-Induced Thermal Acoustics: Simple Accurate Gas Measurements, Opt. Lett. 19 (1994), p. 1361.

[3] E. B. CUMMINGS, H. G. HORNUNG, M. S. BROWN, P. A. DEBARBER, Measurement of Gas-Phase Sound Speed and Thermal Diffusivity Over a Broad Pressure Range Using Laser-Induced Thermal Acoustics, Opt. Lett. 20 (1995), p. 1577.

[4] E. B. CUMMINGS, I. A. LEYVA, H. G. HORNUNG, Laser-induced thermal acoustics (LITA) signals from finite beams, App. Opt. 34 (1995), p. 3290.

[5] A. STAMPANONI-PANARIELLO, B. HEMMERLING, W. HUBSCHMID, Temperature Measurements in Gases Using Laser Induced Electrostrictive Gratings, App. Phys. B 67 (1998), p. 125.

[6] R. C. HART, R. J. BALLA, G. C. HERRING, Nonresonant Referenced Laser-Induced Thermal Acoustics Thermometry in Air, App. Opt. 38 (1999), p. 577. 
[7] R. C. HART, R. J. BALLA, G. C. HERRING, Optical Measurement of the Speed of Sound in Air Over the Temperature Range 300 - 650 K, J. Acoust. Soc. Am. 108 (2000), p. 1946.

[8] M. S. BROWN, W. L. ROBERTS, Single Point Thermometry in High-Pressure Sooting, Premixed Combustion Environments, J. Propulsion and Power 15 (1999), p. 119.

[9] P. F. BARKER, J. H. GRINSTEAD, R. B. MILES, Single-pulse temperature measurement in supersonic air flow with predissociated laser-induced thermal gratings, Opt. Comm. 168 (1999), p. 177.

[10] D. J. W. WALKER, R. B. WILLIAMS, P. EWART, Thermal Grating Velocimetry, Opt. Lett. 23 (1998), p. 1316.

[11] S. SCHLAMP, E. B. CUMMINGS, H. G. HORNUNG, Beam misalignments and fluid velocities in laserinduced thermal acoustics, App. Opt. 38 (1999), p. 5724.

[12] S. SCHLAMP, E. B. CUMMINGS, T. H. SOBOTA, Laser-Induced Thermal-Acoustic Velocimetry with Heterodyne Detection, Opt. Lett. 25 (2000), p. 224.

[13] D. N. KOZLOV, B. HEMMERLING, A. STAMPANONI-PANARIELLO, Measurement of gas jet flow velocities using laser-induced electrostrictive gratings, App. Phys. B 71 (2000), p. 585.

[14] B. HEMMERLING, D. N. KOZLOV, A. STAMPANONI-PANARIELLO, Temperature and flow-velocity measurements by use of laser-induced electrostrictive gratings, Opt. Lett. 25 (2000), p. 1340.

[15] R. C. HART, R. J. BALLA, G. C. HERRING, Simultaneous velocimetry and thermometry of air by use of nonresonant heterodyned laser-induced thermal acoustics, App. Opt. 40 (2001), p. 965.

[16] S. ROZOUVAN, T. DREIER, Polarization-dependent laser-induced grating measurements, Opt. Lett. 24 (1999), p. 1596.

[17] L. E. KINSLER, A. R. FREY, A. B. COPPENS, J. V. SANDERS, Fundamentals of Acoustics, Wiley \& Sons (1982).

[18] A. A. MAZNEV, K. A. NELSON, J. A. ROGERS, Optical Heterodyne Detection of Laser-Induced Gratings, Opt. Lett. 23 (1998), p. 1319.

[19] S. L. MARPLE, Digital Spectral Analysis with Applications, Prentice-Hall (1987). 


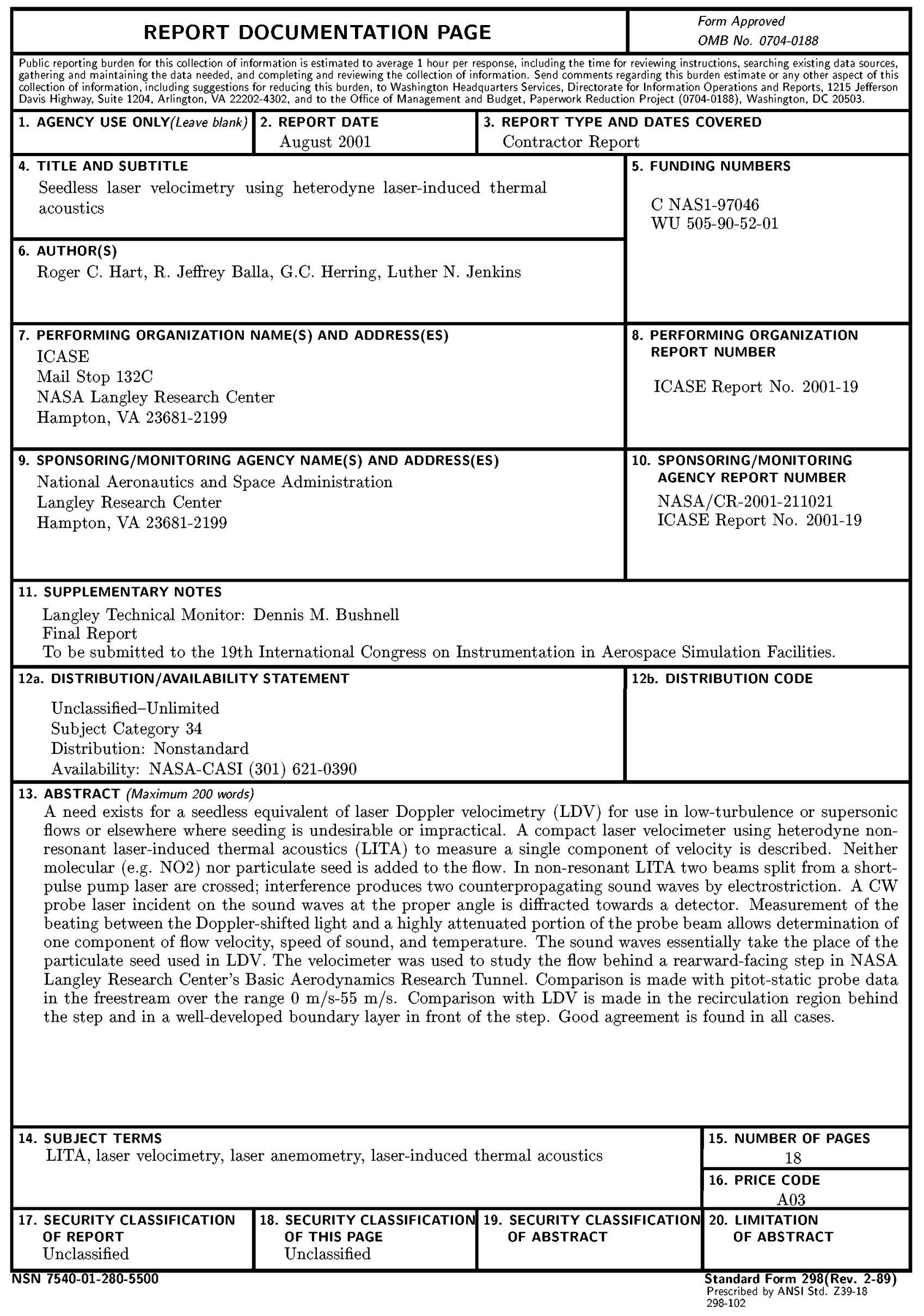

